\title{
LA PARTICIPACIÓN POLÍTICA Y LA CALIDAD INSTITUCIONAL TERRITORIAL EN COLOMBIA*
}

\author{
WILMER JAVIER RÍOS PIÑEREZ** \\ UNIVERSIDAD CATÓLICA DE COLOMBIA
}

Recibido/ Received/ Recebido: 04/12/2013 - Aceptado/ Accepted / Aprovado: 07/05/2014

\begin{abstract}
Resumen
El presente trabajo pretende contrastar la hipótesis de que la participación activa de la comunidad mejora la gestión de los recursos locales. En este sentido, explora si la participación política de los individuos en un ente territorial está relacionada con la calidad de los servicios educativos prestados por dichos entes. Con este objetivo, se ajusta un modelo donde se intenta explicar el auto reporte de satisfacción de los individuos con los servicios educativos prestados por los entes territoriales a partir de la disponibilidad de recursos e índices de participación política local. Se diseñaron dos índices que miden el nivel y la concentración de la participación política por departamentos que se encuentran explicados por variables, que indican el nivel de cultura política y democracia en los individuos, además de variables relacionadas con la disponibilidad de recursos. Los resultados muestran que, además de la disponibilidad de recursos, la concentración de la participación política tiene influencia en la calidad de los servicios educativos prestados por los entes territoriales. Esto apoya la hipótesis de la existencia de una relación sinérgica y no lineal entre la disponibilidad de recursos, las estructuras políticas y la calidad institucional.
\end{abstract}

Palabras clave: Calidad institucional, Desarrollo local, Participación política, Cultura política, Sinergia cognitiva.

\section{POLITICAL PARTICIPATION AND TERRITORIAL INSTITUTIONAL QUALITY IN COLOMBIA}

\begin{abstract}
This paper aims to test the hypothesis that community's active participation improves local resources management. In this sense, the study explores if individual's political participation in a local authority is related to educational services quality provided by such entities. To this end, a model which explains individual's self-reported satisfaction with educational services provided by local authorities is adjusted based on the availability of participation resources and rates of local politics. To measure political participation level and concentration by departments, two indices were design which are explained by variables indicating individuals' political culture and democracy levels and variables
\end{abstract}

Artículo de investigación desarrollado en el proyecto Sinergia Cognitiva y Desarrollo Institucional, dirigido por William Prieto Bustos y financiado por la Universidad Católica de Colombia durante el año 2013. Agradezco a Jorge Andrés Vélez Ospina por sus recomendaciones y comentarios acerca de este trabajo.

* Economista de la Universidad de Cartagena y Magister en Economía de la Universidad de los Andes. Docente investigador de la Facultad de Ciencias Económicas y Administrativas de la Universidad Católica de Colombia. La responsabilidad de los resultados y errores de este estudio recaen totalmente sobre el autor y de ningún modo son responsabilidad de la Universidad Católica de Colombia. Correo electrónico: wjrios@ucatolica.edu.co. 
related to resources availability. The results showed that besides resources availability, the political participation concentration influences educational services quality provided by local authorities. This supports the hypothesis of a synergistic and nonlinear relationship between resources availability, political structures and institutional quality.

Keywords: Institutional quality, Local development, Political participation, Political culture, Cognitive synergy.

\title{
A PARTICIPAÇÃO POLÍTICA E A QUALIDADE INSTITUCIONAL TERRITORIAL NA COLÔMBIA
}

\begin{abstract}
Resumo
O presente trabalho pretende contrastar a hipótese de que a participação ativa da comunidade melhora a gestão dos recursos locais. Neste sentido, explora se a participação política dos indivíduos num ente territorial está relacionada com a qualidade dos serviços educativos prestados por estes entes. Com este objetivo, ajusta-se um modelo onde se tenta explicar o autorrelatório de satisfação dos indivíduos com os serviços educativos prestados pelos entes territoriais a partir da disponibilidade de recursos e índices de participação política local. Desenharam-se dois índices para medir o nível e a concentração da participação política por departamentos, explicados por variáveis que indicam o nível de cultura política e de democracia nos indivíduos, além de variáveis relacionadas com a disponibilidade de recursos. Os resultados mostram que, além da disponibilidade de recursos, a concentração da participação política tem influência na qualidade dos serviços educativos prestados pelos entes territoriais. Isto apoia a hipótese da existência de uma relação sinérgica e não linear entre a disponibilidade de recursos, as estruturas políticas e a qualidade institucional.

Palavras chave: Qualidade institucional, Desenvolvimento local, Participação política, Cultura política, Sinergia cognitiva.

Ríos, W. (2014) La Participación Política y la Calidad Institucional Territorial en Colombia. En: Revista de la Facultad de Ciencias Económicas de la Universidad Militar Nueva Granada. rev.fac.cienc. econ, XXII (1).

JEL: O18, O21, R58.
\end{abstract}

\section{Introducción}

La teoría del crecimiento económico ha ido renovando su interés en la influencia de las instituciones como factor de crecimiento económico. En este sentido, se ha ido encontrando evidencia consistente de la importancia del desarrollo institucional como generador de dinámicas de crecimiento endógeno en las economías. Por otro lado, ha surgido interés en investigar los aspectos territoriales del crecimiento económico, particularmente con Colombia donde existen grandes desigualdades regionales en cuanto a la distribución del crecimiento económico nacional y poca perspectiva de convergencia económica regional. En tal sentido, teniendo en cuenta que el concepto de crecimiento económico endógeno es teóricamente consistente con la dinámica de las estructuras políticas territoriales. Cabe preguntarse si la calidad institucional, medida como la capacidad del Estado para brindar servicios y bienes públicos a los ciudadanos, se encuentra limitada o potenciada no solamente por la disponibilidad de recursos, sino también por el entorno político y, en especial, la participación y organización política de los ciudadanos residentes en los territorios.

Las teorías modernas del crecimiento económico identifican las causas del crecimiento económico con 
las dotaciones de factores productivos y el nivel técnico siguiendo una dinámica de acumulación en el tiempo. A su vez, la promoción del nivel técnico se explica a través de la existencia de marcos institucionales que reducen la incertidumbre, disminuyen los costos de transacción y resuelven los problemas de coordinación social. En este orden de ideas, el crecimiento de largo plazo es explicado por la existencia de instituciones que lo promueven. Los cambios institucionales podrían permitir a economías, en situación de pobreza persistente, generar crecimiento endógeno a partir de saltos en las dinámicas de acumulación de factores producidas por un uso más eficiente de las dotaciones de factores productivos.

Entre los distintos enfoques teóricos que han explicado el desempeño institucional se encuentran los que se enfocan en la capacidad de asumir los costos de transacción que implican las instituciones, por lo que un mayor nivel de actividad económica incentiva la aparición de instituciones favorables (Demsetz, 1967; North, 1981). Otro tipo de teorías tienen que ver con que la redistribución de recursos es diseñada por grupos en el poder $y$, por tanto, la concentración de este facilita a estos capturar recursos y transferirlos a sí mismos (North, 1990). Por otro lado, un grupo de teorías afirma que los rasgos culturales condicionan los mecanismos de acción colectiva (Acemoglu, Johnson \& Robinson, 2001; Acemoglu et al., 2002; Acemoglu \& Johnson, 2005).

La intervención sobre el desempeño institucional tiene consecuencias económicas importantes. El desarrollo institucional podría permitir generar crecimiento económico endógeno a regiones pobres. En tal sentido, la política pública complementaria orientada a impactar variables causantes del mejoramiento institucional podría tener efectos de más largo plazo que solamente la destinación de recursos para la producción de bienes y servicios públicos. Asimismo, el mejoramiento institucional mejora la distribución del ingreso.

Este trabajo se organiza en cinco partes comenzando con la presente introducción. La segunda parte, corresponde a una revisión de literatura empírica acerca de los determinantes de la calidad institucional. La tercera parte es el marco empírico del estudio donde se establece la formulación metodológica y la especificación del modelo empírico. La cuarta parte muestra los resultados y la quinta concluye.

\section{Revisión de literatura}

North (1994) considera que las instituciones son la estructura de incentivos de la sociedad que restringen los mecanismos de ejecución y gobierno influyendo en el comportamiento de los individuos. Los trabajos empíricos han centrado su interés en cómo la calidad de las instituciones afecta el desempeño económico de los países. El resultado característico es que mejores instituciones promueven el crecimiento económico.

Las variables usadas para explicar la eficiencia institucional a nivel de países se pueden clasificar en cuatro grupos (Straub, 2000). El primer grupo consiste en variables históricas o de control; el segundo, son el conjunto de inventivos políticos; el tercero es la existencia de rentas y el cuarto los incentivos burocráticos.

Aixala et al. (2004) y Aixala \& Fabro (2008) toman como variable dependiente la calidad institucional medida con indicadores agregados de gobernabilidad. Sus resultados sugieren que los países pobres etnolingüísticamente heterogéneos, con origen legal francés y socialista y con elevada proporción de católicos y musulmanes, cuentan con inferiores desempeños institucionales. A su vez, sus resultados sugieren que la apertura comercial y la reducción en las desigualdades en renta, contribuirían a mejorar dichos desempeños. Sin embargo, su análisis efectuado por subgrupos de países, clasificados en función del nivel de renta, no ofrece una respuesta causal global en relación a los determinantes de la calidad institucional debido a la existencia de endogeneidades que impiden el análisis causal.

Alonso \& Garcimartín (2011) aportan nuevas evidencias acerca de los factores que determinan la calidad institucional. De acuerdo a sus resultados, son el propio nivel de desarrollo, la equidad en la distribución de la renta, la existencia de un sistema impositivo eficaz y el nivel educativo los principales determinantes de la calidad institucional. Para llegar a es- 
tos resultados utilizan una estimación de los efectos causales mediante variables instrumentales (Straub, 2000) a fin de contrarrestar tres tipo de problemas empíricos: (i) la disposición de unos indicadores de calidad institucional deficientes; (ii) la presencia de endogeneidad en las relaciones estudiadas; (iii) la alta colinealidad entre las variables potencialmente explicativas (que dificulta considerarlas como factores independientes); y (iv) la posible existencia de variables omitidas, que pueden condicionar las relaciones estimadas.

En Colombia, Prieto (2010) vincula el desarrollo territorial con el capital social mediante una aproximación bivariada. El presente trabajo pretende explorar los determinantes de la calidad institucional territorial como variable dependiente y para ello explica el nivel de satisfacción de los individuos con los servicios educativos prestados por los entes territoriales mediante variables que reflejan la riqueza de los entes territoriales (pib per cápita departamental y educación de no mercado), variables que reflejan la cultura política y estructura democrática (nivel y concentración de la participación política por departamento) y variables que reflejan las características socioeconómicas de los individuos.

Este trabajo se diferencia de los anteriormente mencionados de dos maneras. La primera es que la metodología utiliza micro datos para explicar los niveles de satisfacción reportados por los individuos a fin de lidiar con el problema de la doble causalidad en las estimaciones. La segunda es que analiza el desempeño institucional desde el punto de vista territorial de manera similar a Prieto (2010).

\section{Marco empírico}

\subsection{Datos}

Los datos a utilizar son los de los años 2005 a 2011 del Proyecto Latinoamericano de Opinión Pública LAPOP $^{1}$ que realiza un estudio de encuestas sobre cultura política y democracia a ciudadanos colombianos en áreas urbanas y rurales. Esta encuesta también recoge información acerca de las caracterís- ticas socioeconómicas de los individuos. También se utilizan las series de cuentas nacionales departamentales del DANE, en particular las series de producto interno bruto, educación de no mercado y población.

\subsection{Metodología}

\subsubsection{Magnitud y distribución de la participación política territorial}

En este trabajo se realizan dos ejercicios econométricos. En el primero, mediante un modelo probit, se calcula la probabilidad de que un individuo participe de organizaciones políticas. Se incluyen controles por el departamento de residencia y el año, además de un conjunto de variables que recogen características individuales y que se espera que, por si solas, no afecten la calidad de los servicios educativos prestados por el municipio, sino por intermedio de la participación (o no participación) política de los individuos.

De manera más formal, puede expresarse una relación lineal que determina una variable latente $y_{i}^{*}$ tal que:

$$
y_{i}^{*}=X_{i} \beta+\varepsilon_{i}(0)
$$

Donde es una variable no observada tal que, si $y_{i}^{*}>0$ la persona participa al menos una vez al mes en reuniones de algún movimiento o partido político, y si $y_{i}^{*} \leq 0$ el individuo no participa. La variable observada $y^{i}$ toma el valor de 1 cuando el individuo participa y toma el valor de 0 en caso contrario. En tal caso, si $\varepsilon_{i}$ tiene una distribución normal la probabilidad de que un individuo participe en movimientos o partidos políticos es:

$$
P\left(y_{i}=1 / X\right)=P\left(y_{i}^{*}>0 \mid X\right)=F\left(X_{i} \beta\right)
$$

Donde $F\left(X_{i} \beta\right)$ es la distribución normal acumulada evaluada en el valor de la regresión latente para cada individuo. Para el caso particular de este estudio la regresión lineal latente se puede expresar como:

$$
\begin{gathered}
y_{i c t}^{*}=\alpha_{t}+\delta_{c}+X_{c c} \beta+Z_{i c t} \gamma+\varepsilon_{i c t} \\
\text { con } \mathrm{t}=1, \ldots, \mathrm{T} \text { e } \mathrm{i}=1, \ldots, \mathrm{N}(2)
\end{gathered}
$$

$1 \quad$ Las bases de datos y cuestionarios pueden encontrarse en www.lapopsurveys.org. 
Donde $y_{\mathrm{ict}}^{*}$ denota el valor que toma la variable latente para el individuo $i$ en el departamento $c$ en el año $t$. A su vez, $X_{c t}$ contiene características del departamento de residencia que varían en el tiempo y $Z_{\text {ict }}$ son características individuales, tales como la educación, el ingreso y entre otras que inciden sobre la participación política del individuo. Se incluyen variables binarias de año $\alpha_{t} y$ efectos fijos por departamento $\delta_{c}$.

Este ejercicio tiene dos propósitos. Por una parte, intenta capturar la probabilidad de participación en organizaciones políticas de los individuos explicada por variables predeterminadas a las características de los entes territoriales. El segundo propósito de este ejercicio es medir la magnitud de la participación política en un departamento y en un año dados y si esta participación se encuentra concentrada o distribuida entre pocos o muchos individuos a fin de, posteriormente, contrastar la hipótesis de que estas influyen en la calidad de los servicios educativos.

El nivel de participación política por departamentos en un año específico, se halló calculando la media geométrica de las probabilidades predichas para los individuos en cada departamento en un año dado. El nivel de concentración de esta participación se midió calculando el coeficiente de Gini de la participación predicha para los individuos por departamentos $y$ año. El efecto de estas variables sobre el nivel de satisfacción con los servicios educativos se calcula en el segundo ejercicio y es el interés de este estudio.

\subsubsection{El modelo de Probit Ordenado Generalizado}

El segundo ejercicio consiste en estimar un modelo probit ordenado generalizado donde la variable dependiente es el nivel de satisfacción (de 1 a 5) con los servicios educativos prestados por los entes territoriales. Si y es la categoría observada entonces, $\mathrm{y} \in J=\{1,2,3,4,5\}$ donde cada valor denota un nivel de satisfacción dado por las categorías 1: muy malo, 2: malo, 3: ni bueno ni malo, 4: buenos y 5: muy buenos. El probit ordenado permite capturar dos características importantes cuando la variable dependiente es categórica. La primera es que esta toma valores discretos y la segunda es que la escala es ordinal por lo que el cambio de un valor a otro superior no da información de la magnitud del cambio sino solamente del sentido de este.

Formalmente, la variable dependiente categórica observada en cada individuo i tiene una función de densidad latente para cada individuo en un departamento y en el año t. Esta variable latente está ligada a la observación de la variable ordenada y mediante la siguiente regla de observación:

$$
y_{i}=\kappa \operatorname{Si} \tau_{i k}<y_{i} \leq \tau_{(i k+1)}, \text { con } k=1,2, \ldots, \kappa(3)
$$

Donde los umbrales $\tau_{i}$ son estrictamente crecientes por lo que $\tau_{i k}<\tau_{(i k+1}$ para todo $\mathrm{k}$.

La estructura de los datos permite organizar un pseudo-panel probit ordenado. El modelo lineal subyacente al probit ordenado a estimar puede ser representado como:

$$
\begin{gathered}
y_{i}=\mathrm{X}_{\mathrm{i}} \beta+\varepsilon_{i} \\
\operatorname{con} t=1, \ldots, \mathrm{T} \text { e } i=1, \ldots, N(4)
\end{gathered}
$$

La probabilidad acumulada para también está relacionada a un conjunto de variables explicativas. Con $\mathrm{F}$ ( ) siendo la distribución normal estándar acumulada, se tiene que:

$$
\begin{gathered}
\operatorname{Pr}\left[y_{i}=j \mid X\right]=F\left(k_{j}-X_{i} \beta\right) \\
\operatorname{con} j=1,2,3,4,5(5)
\end{gathered}
$$

Por tanto,

$$
\begin{gathered}
y=j \text { si y solo si, } k_{(j-1)} \leq X_{i} \beta+\varepsilon_{i}<k_{j} \\
\text { con } j=1,2, \ldots, 5(6)
\end{gathered}
$$

Lo que significa que los parámetros de umbral dividen la pendiente lineal de y en $J$ categorías. La probabilidad de que un individuo reportará un nivel de satisfacción $J=\{1,2,3,4,5\}$ se expresa como:

$$
\operatorname{Pr}\left[y_{i}=j \mid X\right]=F\left(k_{j}-X_{i} \beta\right)-F\left(k_{(j-1)}-X_{i} \beta\right)(7)
$$

Dado que se asume que el vector no cambia a través de categorías, el incremento de una variable independiente cambia la distribución, pero no su pendiente. A fin de tener una especificación más flexi- 
ble, se definen parámetros de umbral que difieren a través de categorías teniendo un probit ordenado generalizado de forma que:

$$
k_{j}=\tilde{(}\left(k_{j}\right)+X_{i} \gamma_{j}(8)
$$

Donde $\gamma_{j}$ es el parámetro que mide la influencia de las variables explicativas en los umbrales. Por lo tanto,

$$
\operatorname{Pr}\left[y_{i} \leq j \mid X\right]=\mathrm{F}\left(\widetilde{k}_{j}+X_{i} \gamma_{j}-\mathrm{X} \beta\right)=\mathrm{F}\left(\widetilde{k}_{j}-X \beta_{j}\right) \text { (9) }
$$

$X \beta_{j}$ identifica un índice por cada categoría $j$ de la variable de resultado. Quedando generalizado un probit ordenado con J-1 modelos probit binarios.

\subsubsection{Especificación Empírica}

La relación lineal subyacente que se estimará es:

$$
\begin{gathered}
y_{i c t}=\alpha_{t}+\delta_{c}+X_{c t} \beta+Z_{i c t} \gamma+\varepsilon_{i c t} \\
\operatorname{con} t=1, \ldots, \text { T e } i=1, \ldots, N(10)
\end{gathered}
$$

Donde denota el nivel de satisfacción del individuo $i$ con los servicios educativos prestados en el departamento $c$ en el año $t$. Por su parte, $X_{c t}$ contiene características del departamento de residencia que varían en el tiempo y $Z_{\text {ict }}$ son características individuales, tales como la educación, el ingreso, entre otras, que afectan los incentivos a la participación política del individuo. Se incluyen variables binarias de año $\alpha_{t}$ y efectos fijos por departamento $\delta_{c}$. Dentro del vector $X_{c t}$ se encuentra el subconjunto de variables de interés de este estudio; es decir, el nivel y la concentración de la participación política en los departamentos.

\section{Resultados}

En el Tabla 1 se muestran los resultados de la estimación de la probabilidad de que un individuo participe habitualmente de reuniones de algún partido o movimiento político. En el panel izquierdo se muestran los controles para el año y el departamento de residencia. Las categorías omitidas son 2004 y Bo- gotá respectivamente. Es de destacar que el departamento de residencia no tiene ningún efecto significativo sobre la probabilidad de participar evidenciando que, las variables de entorno y cultura democrática determinan más significativamente el nivel de participación política sin importar la localización del individuo.

Por su parte, en el panel derecho se muestran controles de cultura democrática y características individuales. El nivel educativo tiene un efecto positivo en la participación política mientras que el ingreso tiene un efecto negativo, pero insignificante. Ser mujer disminuye las probabilidades de participar; así como no haber sido vulnerable al desplazamiento forzado o víctima de la delincuencia. Este resultado sugiere que las personas vulnerables al desplazamiento forzado y a ser víctimas de la delincuencia tienen incentivos a involucrarse en actividades políticas ya sea para mejorar su acceso a ayuda o como consecuencia del acceso a ella.

Las personas que aprueban la participación colectiva en la solución de los problemas de las comunidades y que otras personas trabajen en las campañas políticas aumentan su propia probabilidad de participar. A su vez, vivir en un departamento más rico o más poblado disminuye las probabilidades de que los individuos se involucren en reuniones políticas, mientras que la existencia de mayores recursos per cápita para brindar educación incentiva el involucramiento en política.

Una vez estimada la probabilidad por individuo se calculan las medidas de participación departamental. En la Ilustración 1 se muestra la evolución de la media aritmética de la probabilidad de participación política por departamentos y la evolución del índice de Gini que mide la concentración de la participación política en los departamentos. En todos los casos, el aumento de la participación política reduce la concentración de esta.

2 Fuente: Cálculos propios usando LAPOP 2006-2011 
Tabla 1. Probabilidad de asistir habitualmente a reuniones de partidos políticos ${ }^{3}$

\begin{tabular}{|c|c|c|c|c|c|}
\hline \\
\hline Variables & \multicolumn{4}{|c|}{$\begin{array}{l}\text { Probabilidad de asistir habitualmente a reuniones partidos políticos } \\
\text { Variables Coeficiente Err. Estándar }\end{array}$} & \\
\hline 2005 & $-0,356^{* *}$ & $(0,109)$ & Mujer & $-0,211^{\star *}$ & $(0,0345)$ \\
\hline 2006 & $-0,321^{\star *}$ & $(0,114)$ & No Victima Delincuencia & $-0,206^{* *}$ & $(0,0695)$ \\
\hline 2007 & 0,136 & $(0,153)$ & No Familiares desplazados & $-0,183^{* *}$ & $(0,0565)$ \\
\hline 2008 & $-0,295$ & $(0,190)$ & No Familiares desplazados fuera del país & $-0,251^{* *}$ & $(0,0739)$ \\
\hline 2009 & $-0,277$ & $(0,177)$ & Con qué firmeza aprobaría que las personas participen & $0,0195+$ & $(0,0105)$ \\
\hline 2010 & $-0,252$ & $(0,225)$ & Con qué firmeza aprobarma o desaprobarma que las $p €$ & $0,0753^{\star *}$ & $(0,0111)$ \\
\hline 2011 & $-0,137$ & $(0,258)$ & Sin Cédula & $-0,516^{* *}$ & $(0,0969)$ \\
\hline Antioquia & $-0,520$ & $(0,393)$ & Años de educación & $0,0318^{* *}$ & $(0,00562)$ \\
\hline Atlántico & $-0,835$ & $(1,936)$ & Ingresos Familiares Mensuales & & \\
\hline Bolivar & $-1,086$ & $(2,264)$ & Menos de $\$ 90.000$ & $-0,252$ & $(0,270)$ \\
\hline Boyacá & $-1,587$ & $(3,017)$ & Entre $\$ 91.000-\$ 180.000$ & $-0,370$ & $(0,263)$ \\
\hline Caldas & $-1,158$ & $(3,376)$ & $181.000-\$ 360.000$ & $-0,311$ & $(0,260)$ \\
\hline Caquetá & $-2,435$ & $(4,801)$ & $361.000-\$ 720.000$ & $-0,319$ & $(0,261)$ \\
\hline Cauca & $-1,316$ & $(2,974)$ & $\$ 721.000-\$ 1.000 .000$ & $-0,276$ & $(0,281)$ \\
\hline Cesar & $-1,214$ & $(3,481)$ & $\$ 1.000 .001-\$ 1.500 .000$ & $-0,417$ & $(0,262)$ \\
\hline Córdoba & $-1,161$ & $(2,691)$ & $\$ 1.500 .001-\$ 2.000 .000$ & $-0,399$ & $(0,305)$ \\
\hline Cundinamarci & $-0,670$ & $(1,879)$ & $\$ 2.000 .001-\$ 3.000 .000$ & $-0,0633$ & $(0,278)$ \\
\hline Huila & $-1,315$ & $(3,284)$ & $\$ 3.000 .001-\$ 4.000 .000$ & $-0,149$ & $(0,237)$ \\
\hline Magdalena & $-1,632$ & $(3,135)$ & $\$ 4.000 .001$ - más & $-0,233$ & $(0,311)$ \\
\hline Meta & $-1,278$ & $(3,686)$ & Características del Departamento & & \\
\hline Nariño & $-1,664$ & $(2,637)$ & Log. PIB percápita depto & $-6,046$ & $(6,836)$ \\
\hline Norte de Sant & $-1,221$ & $(2,976)$ & & 0,186 & $(0,208)$ \\
\hline Quindío & $-1,538$ & $(4,358)$ & Log. población & $-1,689$ & $(1,934)$ \\
\hline Risaralda & $-0,788$ & $(3,479)$ & Log. Educación de no mercado percápita & $1,242+$ & $(0,673)$ \\
\hline Santander & $-0,828$ & $(2,235)$ & Constante & 65,31 & $(69,40)$ \\
\hline Sucre & $-1,731$ & $(3,827)$ & & & \\
\hline Tolima & $-1,304$ & $(2,816)$ & Observations & 9,974 & 9,974 \\
\hline Valle del Cau & $-0,275$ & $(0,869)$ & Pseudo R-cuadrado & 0,103 & 0,103 \\
\hline Casanare & $-2,327$ & $(5,453)$ & Errores estándar clúster por departamentos & & \\
\hline Putumayo & $-2,072$ & $(5,363)$ & ${ }^{* *} p<0.01,{ }^{*} p<0.05,+p<0.1$ & & \\
\hline Vaupés & $-4,015$ & $(8,893)$ & Fuente: Cálculos propios usando LAPOP 2006-2011 & & \\
\hline
\end{tabular}

Los Tablas 2 y 3 presentan los resultados de la estimación del modelo probit ordenado generalizado para el nivel de satisfacción de las necesidades educativas por parte de los entes territoriales. Los coeficientes miden el efecto sobre la probabilidad de que la variable latente supere el umbral hacia la catego- ría superior. Los errores estándar fueron calculados mediante el procedimiento de replicación (bootstrapping) para aproximar la distribución de muestreo de los errores estándar dado que los datos son un pseudo-panel con efectos fijos.

Fuente: Elaboración propia a partir de LAPOP 2005-2011. 
Ilustración 1. Participación política por departamentos ${ }^{4}$

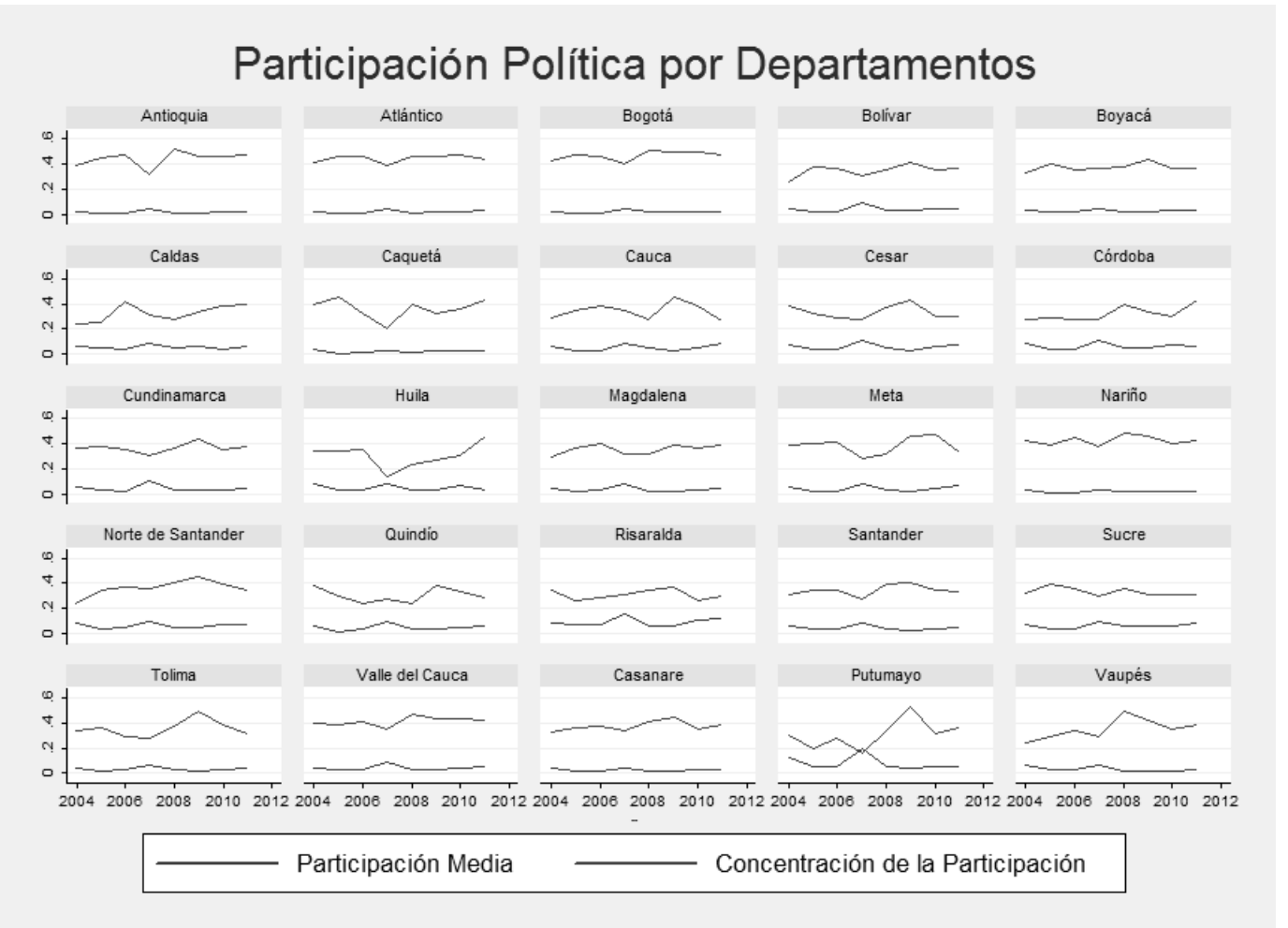

En todos los casos, la cantidad de recursos per cápita que se dedican a la producción de educación de no mercado tiene un impacto significativo sobre la probabilidad de mejorar la satisfacción con los servicios educativos. A su vez, en el Tabla 4 se muestra que el efecto no es lineal y solo es positivo a niveles superiores de satisfacción. El nivel de actividad económica, dado por el PIB per cápita departamental, sólo mejora el nivel de satisfacción cuando este se encuentra en el nivel bueno. La desigualdad en la participación política empeora la calidad de los servicios reversándose el efecto cuando los servicios se encuentran en el nivel muy bueno. Por su parte, el nivel de participación política tiene un efecto negativo cuando la calidad de los servicios es baja y positivo cuando la calidad es alta. Sin embargo ninguno de los efectos es significativo.

Como ejercicio final, se estimó la probabilidad de que los servicios de educación lleguen al nivel "muy bueno" para cada departamento. En el Ilustración 2 se presenta la predicción junto con el índice de Gini de la participación política. La relación negativa se observa solo en algunos casos y débilmente en otros.

4 Fuente: Elaboración propia a partir de LAPOP 2005-2011. 
Tabla 2. Niveles de satisfacción "Muy Malos" y "Malos": Probabilidad de superar el nivel de satisfacción (Modelo probit ordenado $)^{5}$

\begin{tabular}{|c|c|c|c|c|}
\hline \multicolumn{5}{|c|}{ Modelo Probit Ordenado Generalizado } \\
\hline \multirow{2}{*}{ Variables } & \multicolumn{2}{|c|}{ Muy Malos } & \multicolumn{2}{|c|}{ Malos } \\
\hline & Coeficiente & Err. Estándar & Coeficiente & Err. Estándar \\
\hline 2006 & 0,0640 & $(0,292)$ & $-0,0122$ & $(0,110)$ \\
\hline 2007 & $-0,200$ & $(0,490)$ & $-0,225$ & $(0,151)$ \\
\hline 2008 & 0,116 & $(0,557)$ & $-0,183$ & $(0,201)$ \\
\hline 2009 & 0,0383 & $(0,637)$ & $-0,264$ & $(0,218)$ \\
\hline 2010 & $-0,0598$ & $(0,690)$ & $-0,239$ & $(0,229)$ \\
\hline 2011 & $-0,172$ & $(0,768)$ & $-0,275$ & $(0,244)$ \\
\hline Antioquia & 1,611 & $(1,350)$ & 0,208 & $(0,422)$ \\
\hline Atlántico & 8,907 & $(5,805)$ & 2,091 & $(1,871)$ \\
\hline Bolívar & $10,81+$ & $(6,206)$ & 2,242 & $(2,095)$ \\
\hline Boyacá & $14,36+$ & $(8,034)$ & 3,096 & $(2,757)$ \\
\hline Caldas & $20,12^{\star}$ & $(9,438)$ & 4,452 & $(3,218)$ \\
\hline Caquetá & 22,32 & $(13,65)$ & 4,967 & $(4,503)$ \\
\hline Cauca & 13,66 & $(8,751)$ & 2,691 & $(2,787)$ \\
\hline Cesar & $16,76+$ & $(9,234)$ & 4,317 & $(3,249)$ \\
\hline Córdoba & 12,34 & $(7,765)$ & 2,584 & $(2,474)$ \\
\hline Cundinamarca & $8,842+$ & $(5,270)$ & 1,847 & $(1,755)$ \\
\hline Huila & $15,44+$ & $(8,770)$ & 3,591 & $(3,096)$ \\
\hline Magdalena & 14,29 & $(9,070)$ & 2,893 & $(2,934)$ \\
\hline Meta & $18,74^{*}$ & $(9,031)$ & 4,922 & $(3,425)$ \\
\hline Nariño & 12,01 & $(8,017)$ & 2,194 & $(2,464)$ \\
\hline Norte de Santander & $17,66^{*}$ & $(8,558)$ & 3,072 & $(2,806)$ \\
\hline Quindío & $21,19+$ & $(12,01)$ & 5,516 & $(4,045)$ \\
\hline Risaralda & $17,39+$ & $(9,710)$ & 4,515 & $(3,341)$ \\
\hline Santander & $10,97^{*}$ & $(5,591)$ & 2,725 & $(2,034)$ \\
\hline Sucre & $18,45+$ & $(11,07)$ & 3,893 & $(3,576)$ \\
\hline Tolima & $13,11+$ & $(7,950)$ & 2,689 & $(2,646)$ \\
\hline Valle del Cauca & 4,025 & $(2,630)$ & 0,703 & $(0,831)$ \\
\hline Casanare & $30,99^{*}$ & $(13,46)$ & 7,777 & $(5,014)$ \\
\hline Putumayo & $25,55+$ & $(14,91)$ & 6,078 & $(5,040)$ \\
\hline Vaupés & $43,09+$ & $(24,41)$ & 10,95 & $(8,339)$ \\
\hline Rural & 0,0114 & $(0,0883)$ & $-0,0730$ & $(0,0504)$ \\
\hline Prob. Participación Política & $-1,035$ & $(0,967)$ & $-1,214^{*}$ & $(0,516)$ \\
\hline Gini Part política & 1,075 & $(1,323)$ & 0,604 & $(0,629)$ \\
\hline
\end{tabular}

Fuente: Elaboración propia a partir de LAPOP 2005-2011.

6 Fuente: Elaboración propia a partir de LAPOP 2005-2011. 
WILMER JAVIER RÍOS PIÑEREZ

\begin{tabular}{|c|c|c|c|c|}
\hline \multicolumn{4}{|c|}{ Modelo Probit Ordenado Generalizado } \\
\hline \multirow{2}{*}{ Variables } & \multicolumn{2}{|c|}{ Muy Malos } & \multicolumn{2}{c|}{ Malos } \\
\cline { 2 - 5 } & Coeficiente & Err. Estándar & Coeficiente & Err. Estándar \\
\hline Part política media Dpto & 5,144 & $(5,651)$ & 3,192 & $(2,440)$ \\
log pib percápita & 11,61 & $(16,85)$ & 4,423 & $(7,673)$ \\
Ipib_pc2 & $-0,388$ & $(0,523)$ & $-0,157$ & $(0,236)$ \\
logaritmo población & $8,496+$ & $(4,339)$ & 0,917 & $(1,622)$ \\
ed. no mercado percápita & $-0,187$ & $(1,224)$ & $1,609^{*}$ & $(0,743)$ \\
Años de educación & $-0,0144$ & $(0,0104)$ & $-0,0346 * *$ & $(0,00561)$ \\
Constante & $-217,3$ & $(183,2)$ & $-54,66$ & $(71,44)$ \\
Observaciones & 8,532 & 8,532 & \\
\hline Errores estándar por el método de replicación entre paréntesis \\
\hline ** $p<0.01,{ }^{*} p<0.05,+p<0.1$
\end{tabular}

Tabla 3. Niveles de satisfacción "Ni buenos ni Malos" y "Buenos": Probabilidad de superar el nivel de satisfacción (Modelo probit ordenado) ${ }^{6}$

\begin{tabular}{|c|c|c|c|c|}
\hline \multicolumn{5}{|c|}{ Modelo Probit Ordenado Generalizado } \\
\hline Variables & \multicolumn{2}{|c|}{ Ni buenos ni malos } & \multicolumn{2}{c|}{ Buenos } \\
\hline 2006 & Coeficiente & Err. Estándar & Coeficiente & Err. Estándar \\
\hline 2007 & $-0,132+$ & $(0,0781)$ & $-0,0843$ & $(0,136)$ \\
2008 & $-0,247^{*}$ & $(0,0997)$ & $-0,164$ & $(0,183)$ \\
2009 & $-0,201+$ & $(0,118)$ & $-0,264$ & $(0,232)$ \\
2010 & $-0,235+$ & $(0,131)$ & $-0,200$ & $(0,230)$ \\
2011 & $-0,331^{*}$ & $(0,145)$ & $-0,213$ & $(0,259)$ \\
Antioquia & $-0,388^{*}$ & $(0,163)$ & $-0,340$ & $(0,302)$ \\
Atlántico & 0,127 & $(0,326)$ & $-1,127^{*}$ & $(0,573)$ \\
Bolívar & $-0,00940$ & $(1,658)$ & $-5,689+$ & $(3,144)$ \\
Boyacá & $-0,470$ & $(1,885)$ & $-7,100^{*}$ & $(3,594)$ \\
Caldas & $-0,929$ & $(2,528)$ & $-10,06^{*}$ & $(4,802)$ \\
Caquetá & 0,184 & $(2,883)$ & $-9,471+$ & $(5,412)$ \\
Cauca & $-0,724$ & $(4,108)$ & $-18,87^{*}$ & $(7,834)$ \\
Cesar & $-1,065$ & $(2,509)$ & $-10,18^{*}$ & $(4,842)$ \\
Córdoba & $-0,172$ & $(2,936)$ & $-10,74+$ & $(5,547)$ \\
Cundinamarca & $-0,663$ & $(2,249)$ & $-9,327^{*}$ & $(4,317)$ \\
Huila & $-0,451$ & $(1,589)$ & $-5,949^{*}$ & $(3,029)$ \\
Magdalena & $-0,467$ & $(2,814)$ & $-10,50+$ & $(5,856)$ \\
Meta & $-0,922$ & $(2,653)$ & $-10,49^{*}$ & $(5,110)$ \\
& $-0,129$ & $(3,119)$ & $-10,67+$ & $(5,893)$ \\
\hline
\end{tabular}

6 Fuente: Elaboración propia a partir de LAPOP 2005-2011. 
LA PARTICIPACIÓN POLÍTICA Y LA CALIDAD INSTITUCIONAL TERRITORIAL EN COLOMBIA

\begin{tabular}{|c|c|c|c|c|}
\hline \multicolumn{5}{|c|}{ Modelo Probit Ordenado Generalizado } \\
\hline \multirow[t]{2}{*}{ Variables } & \multicolumn{2}{|c|}{ Ni buenos ni malos } & \multicolumn{2}{|c|}{ Buenos } \\
\hline & Coeficiente & Err. Estándar & Coeficiente & Err. Estándar \\
\hline Nariño & $-0,656$ & $(2,251)$ & $-9,120^{*}$ & $(4,301)$ \\
\hline Norte de Santander & $-0,533$ & $(2,546)$ & $-9,824^{*}$ & $(4,782)$ \\
\hline Quindío & 0,0517 & $(3,697)$ & $-12,55+$ & $(7,087)$ \\
\hline Risaralda & 0,314 & $(2,953)$ & $-9,936+$ & $(5,541)$ \\
\hline Santander & $-0,322$ & $(1,844)$ & $-6,813+$ & $(3,587)$ \\
\hline Sucre & $-0,688$ & $(3,239)$ & $-12,45^{\star}$ & $(6,176)$ \\
\hline Tolima & $-0,754$ & $(2,394)$ & $-8,891^{*}$ & $(4,508)$ \\
\hline Valle del Cauca & $-0,166$ & $(0,730)$ & $-2,986^{*}$ & $(1,366)$ \\
\hline Casanare & 0,000383 & $(4,572)$ & $-16,57+$ & $(8,504)$ \\
\hline Putumayo & $-0,759$ & $(4,513)$ & $-16,82^{*}$ & $(8,451)$ \\
\hline Vaupés & $-0,568$ & $(7,571)$ & $-26,94+$ & $(14,54)$ \\
\hline Rural & $-0,0396$ & $(0,0361)$ & $-0,00302$ & $(0,0478)$ \\
\hline Prob. Participación Política & $-0,245$ & $(0,412)$ & 0,535 & $(0,724)$ \\
\hline Gini Part política & 0,281 & $(0,370)$ & $1,609^{*}$ & $(0,745)$ \\
\hline Part política media Dpto & 2,250 & $(1,649)$ & 2,119 & $(3,170)$ \\
\hline log pib percápita & 0,0977 & $(5,072)$ & $-19,66+$ & $(11,27)$ \\
\hline Ipib_pc2 & $-0,00629$ & $(0,156)$ & $0,605+$ & $(0,345)$ \\
\hline Iogaritmo población & $-1,632$ & $(1,522)$ & $-6,835^{\star}$ & $(2,937)$ \\
\hline ed. no mercado percápita & $1,915^{\star \star}$ & $(0,453)$ & $2,434^{\star *}$ & $(0,730)$ \\
\hline Años de educación & $-0,0260^{\star *}$ & $(0,00435)$ & $0,0157^{*}$ & $(0,00611)$ \\
\hline Constante & 12,81 & $(54,71)$ & $248,2^{*}$ & $(120,9)$ \\
\hline Observaciones & 8,532 & & 8,532 & \\
\hline \multicolumn{5}{|c|}{ Errores estándar por el método de replicación entre paréntesis } \\
\hline${ }^{* *} p<0.01,{ }^{*} p<0.05,+p$ & & & & \\
\hline
\end{tabular}

7 Fuente: Elaboración propia a partir de LAPOP 2005-2011. 
Tabla 4. Efectos marginales medios sobre la probabilidad de superar los niveles de satisfacción para cada categoría ${ }^{6}$

\begin{tabular}{|c|c|c|c|c|c|}
\hline \multicolumn{6}{|c|}{ Efectos marginales medios sobre la probabilidad } \\
\hline Variables & Muy Malos & Malos & Ni bs/malos & Buenos & Muy Buenos \\
\hline 2006 & $-0,00155$ & 0,00358 & 0,0482 & $-0,0430$ & $-0,00723$ \\
\hline 2007 & 0,00609 & 0,0352 & 0,0534 & $-0,0813$ & $-0,0134$ \\
\hline 2008 & $-0,00270$ & 0,0357 & 0,0437 & $-0,0563$ & $-0,0203$ \\
\hline 2009 & $-0,000952$ & 0,0502 & 0,0405 & $-0,0737$ & $-0,0160$ \\
\hline 2010 & 0,00161 & 0,0426 & 0,0831 & $-0,110$ & $-0,0170$ \\
\hline 2011 & 0,00511 & 0,0465 & 0,0985 & $-0,125$ & $-0,0250$ \\
\hline Antioquia & $-0,0146$ & $-0,0162$ & $-0,0158$ & 0,0974 & $-0,0508$ \\
\hline Atlántico & $-0,0223$ & $-0,0833$ & 0,109 & 0,0620 & $-0,0656$ \\
\hline Bolívar & $-0,0303$ & $-0,0788$ & 0,293 & $-0,106$ & $-0,0783$ \\
\hline Boyacá & $-0,0566$ & $-0,0658$ & 0,480 & $-0,240$ & $-0,117$ \\
\hline Caldas & $-0,0348$ & $-0,0784$ & 0,0470 & 0,136 & $-0,0702$ \\
\hline Caquetá & $-0,0173$ & $-0,0838$ & 0,384 & $-0,219$ & $-0,0632$ \\
\hline Cauca & $-0,0261$ & $-0,0799$ & 0,508 & $-0,325$ & $-0,0773$ \\
\hline Cesar & $-0,0509$ & $-0,0752$ & 0,192 & 0,0363 & $-0,102$ \\
\hline Córdoba & $-0,0229$ & $-0,0819$ & 0,364 & $-0,188$ & $-0,0719$ \\
\hline Cundinamarca & $-0,0180$ & $-0,0822$ & 0,277 & $-0,117$ & $-0,0597$ \\
\hline Huila & $-0,0127$ & $-0,0838$ & 0,279 & $-0,133$ & $-0,0494$ \\
\hline Magdalena & $-0,0261$ & $-0,0803$ & 0,461 & $-0,278$ & $-0,0767$ \\
\hline Meta & $-0,0318$ & $-0,0836$ & 0,164 & 0,0250 & $-0,0742$ \\
\hline Nariño & $-0,0295$ & $-0,0770$ & 0,363 & $-0,173$ & $-0,0841$ \\
\hline Norte de Santander & $-0,0205$ & $-0,0807$ & 0,310 & $-0,149$ & $-0,0598$ \\
\hline Quindío & $-0,0149$ & $-0,0850$ & 0,0808 & 0,0716 & $-0,0524$ \\
\hline Risaralda & $-0,0645$ & $-0,0684$ & 0,0232 & 0,215 & $-0,105$ \\
\hline Santander & $-0,0313$ & $-0,0823$ & 0,238 & $-0,0477$ & $-0,0772$ \\
\hline Sucre & $-0,0487$ & $-0,0699$ & 0,387 & $-0,165$ & $-0,104$ \\
\hline Tolima & $-0,0371$ & $-0,0755$ & 0,407 & $-0,205$ & $-0,0893$ \\
\hline Valle del Cauca & $-0,0245$ & $-0,0544$ & 0,142 & 0,0109 & $-0,0741$ \\
\hline Casanare & $-0,0387$ & $-0,0799$ & 0,118 & 0,0788 & $-0,0786$ \\
\hline Putumayo & $-0,0228$ & $-0,0838$ & 0,402 & $-0,228$ & $-0,0673$ \\
\hline Vaupés & $-0,0365$ & $-0,0811$ & 0,340 & $-0,139$ & $-0,0840$ \\
\hline
\end{tabular}




\begin{tabular}{|c|c|c|c|c|c|}
\hline \multicolumn{7}{|c|}{ Efectos marginales medios sobre la probabilidad } \\
\hline Variables & Muy Malos & Malos & Ni bs/malos & Buenos & Muy Buenos \\
\hline Rural & $-0,000291$ & 0,0127 & 0,00245 & $-0,0145$ & $-0,000273$ \\
Prob. Participación Política & 0,0265 & 0,174 & $-0,109$ & $-0,140$ & 0,0483 \\
Gini Part política & $-0,0276$ & $-0,0723$ & $-0,00506$ & $-0,0405$ & 0,145 \\
Part política media Dpto & $-0,132$ & $-0,396$ & $-0,312$ & 0,648 & 0,192 \\
log pib percápita & $-0,298$ & $-0,434$ & 0,695 & 1,814 & $-1,777$ \\
Ipib_pc2 & 0,00994 & 0,0161 & $-0,0237$ & $-0,0570$ & 0,0547 \\
logaritmo población & $-0,218$ & 0,0660 & 0,761 & 0,00882 & $-0,618$ \\
ed. no mercado percápita & 0,00480 & $-0,271$ & $-0,449$ & 0,495 & 0,220 \\
Años de educación & 0,000370 & 0,00536 & 0,00396 & $-0,0111$ & 0,00142 \\
\hline
\end{tabular}

Ilustración 2. Calidad de los Servicios Educativos por Departamentos ${ }^{7}$

\section{Calidad de los Servicios Educativos por Departamentos}

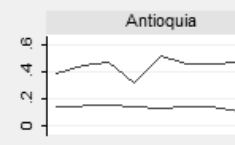

Caldas

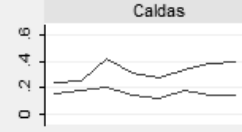

Cundinamarca

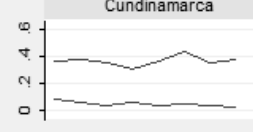

Norte de Santander

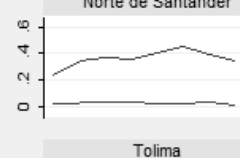

Tolima

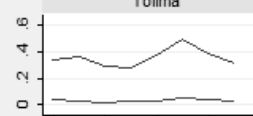

$200420062008201020122004 \quad 2006 \quad 2008 \quad 2010 \quad 2012$

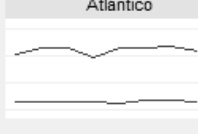

Caquetá

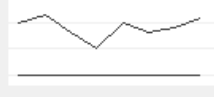

Huila

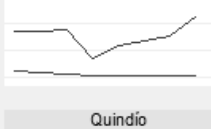

Quindio

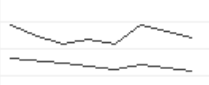

Valle del Cauca
Bogotá

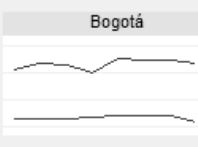

Cauca

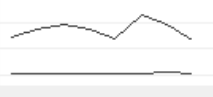

Magdalena

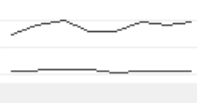

Risaralda

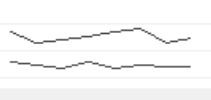

Casanare
Bolivar

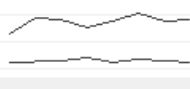

Cesar

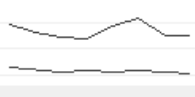

Meta

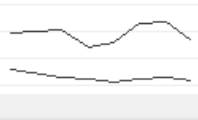

Santander

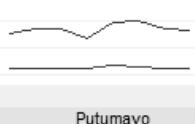

Putumayo

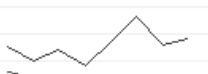

Vaupés

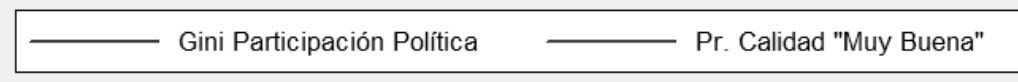




\section{Conclusiones}

La relación entre cultura política, participación democrática y la calidad de los servicios brindados por los entes territoriales no está bien establecida. La evidencia encontrada sugiere que no siempre la disponibilidad de recursos conlleva mejoras institucionales. Asimismo, se da para el aumento del acceso a la participación política. En tal sentido, lo que sí se puede concluir es que existen complementariedades y sinergias entre disponibilidad de recursos, el nivel de participación política y el acceso a ésta. Cuando los servicios educativos alcanzan un nivel aceptable de calidad, la disponibilidad de recursos y el acceso más igualitario a la participación política permite aumentar significativamente la calidad de los servicios.

La baja calidad de los servicios educativos de los entes territoriales está relacionada con carencia de recursos y concentración de la participación política en pocos individuos. No obstante, para estos departamentos, el aumento en los recursos disponibles no implica una mejora significativa en la calidad de la prestación de servicios educativos. Sino por el contrario, tiene un impacto negativo aunque no significativo.

Una posible explicación es la existencia de fenómenos de captura del Estado, búsqueda de rentas y corrupción. En tal sentido, hay evidencia de que las personas ya sea víctimas de delitos o cuya familia ha padecido desplazamiento forzado tienen más probabilidades de participar en reuniones políticas. Este resultado sugiere que la mayor demanda individual de servicios gubernamentales se relaciona con vulnerabilidades que inducen a los individuos a asociarse a grupos con el fin de gestionar recursos públicos.

Futuras aproximaciones a la calidad institucional territorial tienen el reto de incorporar un análisis dinámico de largo plazo e incorporar nueva información que permita tener variables de exclusión que permitan aislar empíricamente los efectos causales de la cultura política y el ejercicio democrático en la gestión de los recursos.

\section{Referencias}

Acemoglu, D. \& Johnson, S. (2005). Unbundling Institutions. En: Journal of Political Economics: 949-995.

Acemoglu, D., Johnson, S. \& Robinson, J. (2001). Colonial Origins of Comparative Development: an Empirical Investigation. En: Quarterly Journal of Economics: 1231-1294.

Acemoglu, D., Johson, S. \& Robinson, J. (2002). Reversal of Fortune: Geography and Institutions in the Making of the Modern Worl Income Distribution. En: Quarterly Journal of Economics: 1231-94.

Acemoglu, D., Jonson, S. \& Robinson, J. (2004). Institutions as the Fundamental Cause of Long-Run Growth. Cambridge: National Boureau of Economic Research.

Aixala, J. \& Fabro, G. (2008). Determinantes de la Calidad Institucional de los Países. En: Revista de Economía Aplicada, 16(46).

Aixala, J., Fabro, G. \& Simón, B. (2004). Calidad Institucional y Factores Político-Culturales: Un Panorama Internacional por Niveles de Renta. Documento de Trabajo. Universidad De zaragoza.

Alonso, J. \& Garcimartin, C. (2011). Criteiros y Factores de Calidad Institucional: Un Estudio Empírico. En: Revista de Economía Aplicada, XIX(55).

Departamento Administrativo Nacional de Estadística DANE. (30 de Junio de 2013). Obtenido de DANE: https://www.dane.gov. co/index.php/cuentas-economicas/cuentas-departamentales.

Demsetz, H. (1967). Towards a Theory of Property Rights. En: The American Economic Review, 57(2): 347-359.

Latin American Political Opinion Project. (31 de Enero de 2013). Obtenido de LAPOP Free Data Acces: http://datasets.americasbarometer.org/database-login/usersearch.php

North, D. (1981). Growth and Structural Change. New York: Norton.

North, D. (1990). Institutions, Institutional Change and Economic Performance. Cambridge University Press.

North, D. (1994). Economic Performance Through Time. En: The American Economic Review, 84(3): 359-368.

Prieto, W. (2010). Sinergia Cognitiva y Pobreza. Universidad Católica de Colombia, Facultad de Economía.

Prieto, W. (2010). Sinergia Cognitiva y Pobreza. Universidad Católica de Colombia, Facultad de Economía.

Straub, S. (2000). Empirical Determinants or Good Institutions. Working Paper. Banco Interamericano de Desarrollo, Departamento de Investigación. 